\title{
A Worksite Health-Monitoring Program: Effectiveness on Detection, Intent to be treated and Follow up Care for Cardiovascular Diseases/Risk Factors
}

\author{
By Thomas A. Mackey*
}

\begin{abstract}
The study objective was to determine the effectiveness of a workplace health-monitoring program on the detection, intent to be treated and follow up care related to multiple cardiovascular diseases (CVD) and risk factors. Environmental investigators for the State of Texas are required to undergo annual health-monitoring exams conducted yearly with intent to determine fitness for duty and detection of work related illnesses. Exams include: work and personal health histories, vital signs, chest $X$ ray, spirometry, electrocardiography, audiometry, blood chemistries, workplace appropriate immunizations, and physical examination. Employees receive personal follow up consultation four weeks post screening. Personal versus work related health problems were difficult to discern. Relatively few occupationally related health problems were detected during monitoring exams. However, a minor percentage of employees were found to have other health conditions significant enough to be restricted from engaging in one or more work-related activity (i.e. respirator use, climbing). However, 20 years of experience examining employees for the same employer has shown significant numbers diagnosed with new CVD and/or risk factors (hypertension, obesity, diabetes, abnormal EKGs, hyperlipidemia, sedentary life style) during the health-monitoring exams. Furthermore, experience indicated a relatively high percentage of employees dropped out of previous treatment for a CVD condition or failed to return for follow up care to a primary care provider/cardiologist. After undergoing an exam and follow up consultation during the health- monitoring program sizeable numbers of employees indicated an intent to start, return to treatment and/or adopt life-style changes to address one or more CVD problem or risk factor. Data from the 2017/19 programs quantify past experience and provides statistics on which to build future interventions. The described health-monitoring program detected meaningful numbers of employees with known/unknown CVD and risk factors. Brief counseling during the physical examinations and follow-up counseling sessions were pivotal in moving employees to indicate intent to re-enter or begin treatment and life-style changes.
\end{abstract}

Keywords: Worksite health-monitoring program, Cardiovascular risk factors, Health-monitoring follow up

\section{Introduction}

Modifiable cardiovascular disease (CVD) risk factors are significant indicators of future chronic health problems. Once CVD becomes a personal health issue the social and economic consequences can magnify leading to loss workdays, financial problems, comorbidities, and eventual deteriorating health status. However, the downward spiraling health status scenario can be modified through early screening, detection and treatment. In recent years screening for CVD risk factors (obesity, tobacco use, elevated blood pressure, diabetes, hyperlipidemia, and sedentary lifestyle) has intensified and provided added value to the workplace health screening and disease prevention movement. In 2014 the American Heart Association (AHA) published rationale, guidelines and a policy on the role of worksite screening for CVD risk factors (Arena et al. 2014).

${ }^{*}$ Professor, University of Texas Health Science Center at Houston, USA 
The current study was based on the AHA recommendations and designed to determine the effectiveness of a workplace health-monitoring program on the detection, intent to be treated and follow up care related to multiple cardiovascular diseases (CVD) and risk factors over a three period of time. Twice, at the same worksites, during the three-year period data were collected and employees offered follow up consultation. Findings indicate significant changes in health behaviors and add credence to the literature regarding the importance of worksite CVD risk screening.

\section{Literature Review}

Oakes (2018) and the Centers for Disease Control and Prevention (CDC) ${ }^{1}$ report CVD remains the leading cause of mortality in the United States. The most recent updated 2019 data indicates 116.4 million, or $46 \%$ of US adults are estimated to have hypertension (Benjamin et al. 2019). The findings are documented in the new 2017 Hypertension Clinical Practice Guidelines (Benjamin et al. 2019).

Identification combined with subsequent intervention directed toward modifiable CVD risk factors (obesity, diabetes, sedentary life style, elevated blood pressure, smoking/tobacco use and hyperlipidemia) are crucial if impact on health and healthcare costs is to improve (Whelton et al. 2017). The workplace presents a unique and ideal setting to discover, intervene and offer assistance to individuals with modifiable CVD risk factors. In fact, some work settings require annual health-monitoring as mandated by the Occupational Safety and Health Administration (OSHA) ${ }^{2}$. Since 1970, OSHA has assured safe and healthful work conditions for employed men and women by establishing and enforcing standards and by providing training, outreach, education and assistance OSHA's mission is to prevent workplace deaths, injuries and illnesses and accomplishes the mission by requiring pre-employment screenings (medical and occupational history, physical examination, determination of fitness to work wearing protective equipment and baseline monitoring for specific exposures). OSHA requires periodic screenings inclusive of yearly update of medical and occupational history; physical examination; testing based on examination results, job class and task, and more frequent and directed testing based on specific exposures.

Regardless, mandated screenings alone do little to change behavioral patterns for individuals with CVD risk factors. However, the addition of follow-up counseling and organizational support programs have proven successful in changing employee CVD risk factors. For example, Soler et al. (2009) evaluated 86 studies of health screening programs some of which included and others excluded follow up consultations with employees. Groups including follow up consultations showed favorable CVD risk factor improvement: some

${ }^{1}$ Center for Disease Control and Prevention. Retrieved from https://bit.ly/2EDMLBD.

${ }^{2}$ OSHA recommended worksite screening. Retrieved from https://ces.to/kzkbPd \& OSHA Guidelines. Retrieved from https://ces.to/IgOOJM. 
increase in smoking cessation, less saturated fat intake, additional physical activity, and enhanced fruit and vegetable intake.

Colkesen et al. (2011) reported a 17.9\% improvement among 368 workers' Framingham Risk Score (FRS) receiving a Web-based health risk assessment coupled with post screening consultation. Additionally, Hochart and Lang (2011) showed positive results when employees consulted with an advisor after completing a program screening. Almost $49 \%$ of high-risk individuals improved risk status and $40 \%$ of the lower risk employees showed improvement. Rula and Hobgood (2010) evaluated over 5,000 employees who completed the myhealthIQ health-screening program. Compared to non-counseled participants high-risk employees who received additional counseling showed improvements in lipid levels (-3.9 to $-6.1 \%)$, smoking cessation $(-2.5 \%)$ and diastolic blood pressure ($3.4 \%)$.

While costly, wellness and health-monitoring programs have the potential of being a positive financial return for employers. Naydeck et al. (2008) studied the financial impact of an employee wellness program over a four-year period of time. For employees who received counseling after screening "multivariate models estimated health care expenses per person per year as \$176 lower for participants. Inpatient expenses were lower by $\$ 182$. Four-year savings of $\$ 1,335,524$ compared with program expenses of $\$ 808,403$ yielded an ROI of $\$ 1.65$ for every dollar spent on the program". For employees not receiving post screening counseling there were no associated health care dollar cost savings. Figure 1 calculations are based on Naydeck's research.

\section{Methodology}

Environmental investigators for the State of Texas are required to undergo annual health-monitoring exams. Prior to the year 2001 employees from all over the State of Texas traveled to Houston for the annual exam incurring costs for air tickets, rent cars, lodging, meals, parking, and at least one lost workday. Rather than employees traveling to Houston, in 2001 exams were delivered on-site in 15 different cities throughout the State of Texas with the employer not incurring the previous mentioned expenses (Figure 1). Employees ranged in age from 22 to 71, have full health insurance through the State of Texas, incomes range between $\$ 35,000$ to $\$ 75,000$ and everyone was/is employed on a full time basis. The exams (males 54\% and females $46 \%$ ) were conducted yearly with intent to determine fitness for duty and detection of undiagnosed work related illnesses. In 2017, 586 evaluations were conducted while in 2018 no CVD risk data were collected on the study population. However, in 2019 data were once again gathered on 601 employees. Exams included: complete work and personal health history, vital signs, chest X ray, spirometry, electrocardiography, audiometry, laboratory work (complete blood count, 24 chemistries, lipids, cholinesterase and lead levels), workplace appropriate immunizations, and a physical examination. A certified medical assistant determined employee blood pressures via a stethoscope and arm based sphygmomanometer. During the exam a nurse practitioner (NP) completed 
a special assessment tool (Figure 2), separate from the complete history form, designed to capture blood pressure, patient CVD risk factors, last visit to a primary care provider (PCP)/cardiologist, intent to see a $\mathrm{PCP} /$ cardiologist pre/post visit, and intent to change lifestyle pre/post visit. The NP provided brief counseling during the examination directed at moving employees with CVD risk factors to action: exercise, schedule a visit with a primary care provider/cardiologist, quit smoking, change other life-style habits. Employees received personal follow up consultation four weeks post exam once testing results were collated into a multiple page personalized report. Data was gathered over a two-year period of time during the months of January thru April.

Figure 1. Program Worth \& Value Per Year Costs and Return on Investment

\begin{tabular}{|c|c|c|}
\hline \multicolumn{2}{|c|}{ Worth } & \multirow{2}{*}{$\begin{array}{c}\text { Value } \\
\text { New diagnostic findings }\end{array}$} \\
\hline Pre mobile unit cost/year & $\$ 790,130$ & \\
\hline With mobile unit cost/year & $\$ 452,125$ & Significant CVD findings \\
\hline Savings/year & $\$ 338,005$ & Intent to change health behavior \\
\hline \multicolumn{3}{|c|}{ Return on investment (Naydeck et al. 2008) } \\
\hline \$176/employee X 586 & $\$ 103,136$ & \\
\hline
\end{tabular}

Source: Author's estimations and Naydeck et al. (2008).

Figure 2. Cardiovascular Disease Risk Assessment Form

Cardiovascular Disease/Risk Assessment

BP: /

\begin{tabular}{|l|l|l|l|}
\hline Patient risk factor & Yes & New DX & Uncontrolled or worse \\
\hline Hypertension/BP elevated & & & \\
\hline Obesity & & & \\
\hline Diabetes & & & \\
\hline Abnormal EKG & & & \\
\hline Hyperlipidemia & & & \\
\hline Sedentary life-style & & & \\
\hline Smoker & & & \\
\hline
\end{tabular}

\begin{tabular}{|l|l|}
\hline Last visit to PCP/cardiologist & \\
\hline$<1$ month & \\
\hline $1-3$ months & \\
\hline $3-6$ months & \\
\hline $6-12$ months & \\
\hline$>1$ year & \\
\hline
\end{tabular}

\begin{tabular}{|l|l|l|}
\hline $\begin{array}{l}\text { Intent to see/RTC to PCP/cardiologist within } 1 \text { month } \\
\text { Pre consult: }\end{array}$ & Yes & No \\
\hline Post consult: & & \\
\hline
\end{tabular}

\begin{tabular}{|l|l|l|}
\hline $\begin{array}{l}\text { Intent to change life-style } \\
\text { Pre consult: }\end{array}$ & Yes & No \\
\hline Post consult: & & \\
\hline
\end{tabular}

Source: Author's estimations. 
In study year 01 (2017) blood pressure levels above 130/90 were considered elevated. CVD risk data on the study population were not collected in 2018. However, in study year 02 (2019) the blood pressure levels changed to readings above 120/80 as being considered elevated (American College of Cardiology 2019) $)^{3}$. The difference reflects new guidelines published by the American Heart Association (AHA) in mid 2018 for blood pressure classification and diagnosis of hypertension (Whelton et al. 2017). However, of note, according to Crawford (2017) the American Academy of Family Physicians (AAFP) does not endorse the new guidelines. The AAFP reasoning is "according to the AHA, the new threshold would lead to $46 \%$ of the US adult population being categorized as having hypertension. Using the previous threshold, that figure would be $32 \%$ of American adults. Furthermore, the AAFP did not feel the bulk of the guidelines were based on a systematic evidence review. Additionally, although the guideline's recommendations were given an evidence quality grade, they weren't grounded in an assessment of the background resources. Finally, substantial weight was given to the Systolic Blood Pressure Intervention Trial (SPRINT), but other trials were minimized" (Crawford 2017). Moreover, while widely adopted by medical professionals in the United States, the new guidelines are not well embraced in Europe (Williams et al. 2018). Regardless of the AAFP and European (European Society of Cardiology-ESC and the European Society of HypertensionESH) reluctance to accept the new guidelines, the AHA recommendations are widely accepted throughout the United States as a community standard for practice and, therefore, utilized in the present study.

The data collected and exam-associated counseling shadowed the AHA's recommendations for workplace screening (Arena et al. 2014). Counseling actually occurred on two separate occasions. The first counseling happened during the exam when the NP discovered the presence of one or more risk factors. $100 \%$ of employees with a risk factor received a brief counseling intervention by the NP. Four to six weeks post screening the NP made a return visit to each of the 15 facilities to provide general information about the results to large groups of employees followed, if desired, by individual counseling sessions. Over the years an estimated $70 \%$ of employees have availed themselves of the individual counseling option post examination.

\section{Results}

The OSHA mandates health monitoring of employees for specific work related conditions yet relatively few occupationally related problems are detected during monitoring exams. However, only a small percentage of employees were found to have health conditions (cardiovascular, pulmonary, neurological, orthopedic) significant enough to be restricted from engaging in one or more work-related activity (i.e., respirator use, climbing, walking long distances, etc.).

\footnotetext{
${ }^{3}$ American College of Cardiology: New ACC/AHA High Blood Pressure Guidelines Lower Definition of Hypertension. Retrieved from https://bit.ly/2EaIx2N.
} 
Figure 3 summarizes the significant medical findings/benefits of the study. Personal versus work related health problems are often difficult to discern. The study did not attempt to differentiate between the two. However, 20 years of experience providing exams for the same employer indicates significant numbers of employees were diagnosed with new primary care health care problems as well as CVD and/or risk factors (hypertension, obesity, diabetes, abnormal EKGs, hyperlipidemia, sedentary life style) identified in Tables 1, 2, 3, and 4.

Figure 3. Summary of Significant Medical Findings/Benefits

- Multiple new primary care diagnoses (sleep disorders, thyroid tumors, hearing loss, depression)

- Newly diagnosed significantly abnormal EKGs

- Newly diagnosed elevated blood pressures

- Uncontrolled blood pressures

Lipid and glucose abnormalities, known risk factors for CVD, were evaluated on each employee. Table 1 shows no statistical difference in the percent of abnormal values between the study years indicative of no change in CVD health status related to the cholesterol, high density lipoproteins (HDL), low density lipoproteins (LDL), triglycerides and glucose markers.

Table 1. Results: Abnormal Lipids and Glucose

\begin{tabular}{|l|c|c|c|}
\hline Laboratory Measure & Reference Values & $\boldsymbol{\# / \%}$ & Abnormal \\
\hline & & $2017(\mathrm{~N}=586)$ & $2019(\mathrm{~N}=593)$ \\
\hline Cholesterol & $<200$ & $200(34 \%)$ & $198(33 \%)$ \\
\hline HDL & $>39$ & $111(19 \%)$ & $98(17 \%)$ \\
\hline LDL & $<100$ & $323(55 \%)$ & $318(54 \%)$ \\
\hline Triglycerides & $<150$ & $143(24 \%)$ & $139(23 \%)$ \\
\hline Glucose & $<100$ & $114(20 \%)$ & $143(24 \%)$ \\
\hline
\end{tabular}

Source: Author's estimations.

Weight, measured by basal metabolic index (BMI) as shown in Table 2, showed some improvement from year to year. For those with a BMI of $>30$ there was a decrease of 5\% indicating some progress in weight control in the study population. Change in BMI for individuals in the $>30$ category may be a result of the NP's intervention but such a conclusion cannot be determined without further investigation and inquiry.

Table 2. Results: Basal Metabolic Index (BMI)

\begin{tabular}{|l|c|c|}
\hline BMI & $\# / \%$ & Abnormal \\
\hline & $2017(\mathrm{~N}=586)$ & $2019(\mathrm{~N}=601)$ \\
\hline $25-29$ & $169(29 \%)$ & $172(29 \%)$ \\
\hline$>30$ & $212(36 \%)$ & $189(31 \%)$ \\
\hline$>40$ & $40(7 \%)$ & $46(8 \%)$ \\
\hline
\end{tabular}

Source: Author's estimations. 
Table 3 presents the two years of abnormal blood pressure readings, uncontrolled known hypertensives and new diagnosis of blood pressure elevation. In 2018 a normal systolic reading was $<140$ and diastolic <90. However, as previously mentioned, the AHA guidelines changed. In 2019, 51\% (306 individuals) had blood pressure elevations according to the AHA guidelines. Consequently, the percent of abnormal readings rose from 9\%/10\% (systolic/diastolic) to $38 \% / 38 \%$ (systolic/diastolic) from one year to the next. Given the new AHA guidelines the additional 20\% increase in 2019 in abnormal blood pressure readings is to be expected.

Table 3. Results: Abnormal Blood Pressure*

\begin{tabular}{|l|c|c|}
\hline Measure & $\# / \%$ & Abnormal \\
\hline & $2017(\mathrm{~N}=586)$ & $2019(\mathrm{~N}=601)$ \\
\hline Systolic blood pressure & $9 \%(53)$ & $38 \%(227)$ \\
\hline Diastolic blood pressure & $10 \%(60)$ & $38 \%(231)$ \\
\hline Uncontrolled known hypertension** & $2.5 \%(15)$ & $7 \%(41)$ \\
\hline New DX of elevated BP & $5 \%(31)$ & $2.8 \%(17)$ \\
$>140 / 90$ & - & $9 \%(53)$ \\
$>130 / 90$ & - & $11 \%(66)$ \\
$>120 / 80$ & &
\end{tabular}

$* 2017>140 / 90$ and $2019>120 / 80$

** $2017>140 / 90$ and $2019>130 / 90$

Source: Author's estimations.

Other CVD risk factors (smoking, alcohol use of three or more drinks per day, abnormal EKG, sedentary life style, and diabetes as reported by the employees did not significantly change from one year to the next (Table 4). Percent of abnormalities in the measured categories are below those of the general United States population.

Table 4. Results: Other CVD Risk Factors for those with BP Elevations

\begin{tabular}{|l|c|c|}
\hline Measure & $\# / \%$ & Abnormal \\
\hline & $2017(\mathrm{~N}=160)$ & $2019(\mathrm{~N}=306)$ \\
\hline Smoking & $3 \%(20)$ & $3 \%(20)$ \\
\hline ETOH (3+drinks/day) & $1 \%(6)$ & $<1 \%(4)$ \\
\hline Abnormal EKG & $9 \%(52)$ & $<1 \%(10)$ \\
\hline Sedentary life style & $6 \%(35)$ & $29 \%(90)$ \\
\hline Diabetes & $5 \%(29)$ & $13 \%(41)$ \\
\hline
\end{tabular}

Source: Author's estimations.

The main study objective was to determine the effectiveness of a workplace health-monitoring program on the detection, intent to be treated and follow up care related to multiple cardiovascular diseases (CVD) and risk factors. Table 5 presents data on employees with known CVD risks intent to change behavior. Experience with the study population indicates a relatively high percentage of employees dropped out of treatment for a previously diagnosed CVD condition or failed to return for follow up care to a primary care provider/cardiologist. After undergoing an exam and follow up consultation during the health- monitoring 
program significant numbers of employees indicated an intent to start, return to treatment and/or adopt life-style changes to address one or more CVD problem(s) or risk factor(s) (Table 5).

Table 5. Results: Employees with Known CVD Risks Intent to Change from "No" to "Yes" Pre/Post Consultation

\begin{tabular}{|l|c|c|}
\hline Intent & $\mathbf{2 0 1 7}(\mathbf{N}=\mathbf{1 6 0})$ & $\mathbf{2 0 1 9}\left(\mathbf{N}=\mathbf{3 0 6}^{*}\right)$ \\
\hline To see PCP/cardiologist within one month & $42 \%(67)$ & $18 \%(55)^{* *}$ \\
\hline To change lifestyle & $20 \%(33)$ & $24 \%(72)^{* *}$ \\
\hline
\end{tabular}

* Larger $\mathrm{N}$ due to change in BP elevation guidelines by AHA.

** Many employees with BPs minimally elevated not needing PCP follow up care.

Source: Author's estimations.

\section{Discussion}

The current study was an attempt to translate research into practice. Ample literature indicates workplace screening is helpful to address CVD risk factors. Nevertheless, screening alone, without follow-up counseling, provides little improvement on health status and does not offer a positive financial return on investment. "However, most evidence evaluating the effectiveness of worksite health screening demonstrates that the most effective model is one that combines screening with encouragement to participate in some type of health and wellness program" (Arena et al. 2014).

How does intent to change health behavior translate into actual change? The program described did not measure actual behavior change but only intent. Future studies on the same population would be of value to determine the real effect of the NP intervention consisting of suggesting and discussing behavior change (i.e., go see a provider or change a life-style). So, while helpful, the study leaves us short on actual behavior change. Regardless, based on the literature presented, some behavioral change probably did/does occur as the study population showed intent to change behavior. Follow-up studies could measure and quantify actual change and the effects on individual CVD risk factors.

What do we know about the blood pressure measurement accuracy and findings for the current study? Blood pressure measurement is a standard for diagnosing hypertension, a CVD risk factor. Measurement in the general population is wrought with potential errors including variability of operator hearing, vision and recording; accurate calibration of the sphygmomanometer, patient preparation, and height of the patient's arm relative to the heart (Muntner et al. 2019). The present study relied on readings recorded by a certified medical technician performing the procedure with a stethoscope and arm based sphygmomanometer. Given recent recommendations by Roerecke et al. (2019) blood pressure readings via an automated office blood pressure (AOBP) monitor is the preferred method due to accuracy and reduction of the "white coat syndrome". "Based on the evidence, AOBP should now be the preferred method for recording BP in routine clinical practice" (Roerecke et al. 2019). Future studies such as the one described herein would benefit from using the AOPB 
monitor to improve accuracy in determining if a patient meets the criteria for elevated blood pressure, or hypertension, as a CVD risk factor. Regardless, the method used was auscultation and results indicate significant numbers of employees with elevated and/or uncontrolled/untreated hypertension. Of particular note is the increase from 2017 to 2019 of uncontrolled known hypertension (Table 3 ) from $2.5 \%$ to $7 \%$ with no readily apparent epidemiological reason.

Is there an accurate tool available to measure CVD risk factors during health screenings? Figure 2 depicts the CVD Risk Assessment Form utilized in the study. When the current study began there was "no tool to assess the effectiveness of the programme in communicating CVD risk to patients" (Woringer et al. 2017). However, in 2017 Woringer et al. (2017) developed such a tool. "The 26-item questionnaire constitutes four scales: Knowledge of CVD Risk and Prevention, Perceived Risk of Heart Attack/Stroke, Perceived Benefits and Intention to Change Behavior and Healthy Eating Intentions" (Woringer et al. 2017). If patients were willing to complete a 26-item questionnaire, future studies to assess CVD risk would benefit from using the tool developed by Woringer et al. (2017) already tested for reliability and validity.

\section{Conclusion}

The described health-monitoring program translates research into practice and detected significant numbers of employees with known/unknown CVD and risk factors. While the current study does not discern the value of follow up counseling, the literature indicates follow-up counseling sessions to be pivotal in moving employees to re-enter or begin treatment and life-style changes. Further studies on the current population to determine if follow up visits/counseling is effective would be useful.

Value and worth of medical screening is a point of great discussion in the literature. Worth refers to money generated or saved. Value refers to money plus intangibles, such as quality, savings, and goodwill (Mackey 2009), new diagnosis, newly discovered CVD risk factors and employees' intent to change behavior. Figure 1 presents pre/post mobile unit costs with a yearly savings of $\$ 338,005$ plus an ROI of over $\$ 100,000$ per year. The real program value, however, comes in the discovery of multiple new primary care diagnoses, CVD risk factor detection and employees intending to change health behaviors. Two years of program data quantifies past experience and provides statistics on which to build future interventions and evaluations.

\section{References}

Arena R, Arnett DK, Terry PE, Li S, Isaac F, Mosca L, et al. (2014) The role of worksite health screening: A policy statement from the American Heart Association. Circulation 130(8): 719-734. 
Benjamin E, Muntner P, Alonso A, Bittencourt M, Calloway C, Carson AP, et al. (2019) Heart disease and stroke statistics - 2019 update: A report from the American Heart Association. Circulation 139(10): 56-528.

Colkesen EB, Ferket BS, Tijssen JG, Kraaijenhagen RA, van Kalken CK, Peters RJ (2011) Effects on cardiovascular disease risk of a web-based health risk assessment with tailored health advice: a follow-up study. Vascular Health Risk Management (7): 67-74.

Crawford C (2017) AAFP decides to not endorse AHA-ACC hypertension guidelines. Retrieved from https://bit.ly/2zaPmzb.

Hochart C, Lang M (2011) Impact of a comprehensive worksite wellness program on health risk, utilization, and health care costs. Popular Health Management 14(3): 111-116.

Mackey T (2009) Practice value and worth of a DNP. Clinical Scholars Review 2(1): 8-9.

Muntner P, Shimbo D, Carey R, Charleston J, Gaillard T, Misra S, et al. (2019) Measurement of blood pressure in humans: A scientific statement from the American Heart Association. Hypertension 73(5): 35-66.

Naydeck BL, Pearson JA, Ozminkowski RJ, Day BT, Goetzel RZ (2008) The impact of the Highmark employee wellness programs on 4-year healthcare costs. Journal of Occupational and Environmental Medicine 50(2):146-156.

Oakes K (2018) Heart disease remains the leading cause of death in the US. Clinicians Review. Retrieved from https://bit.ly/2vXjEmM.

Roerecke M, Kaczorowski J, Myers M (2019) Comparing automated office blood pressure readings with other methods of blood pressure measurement for identifying patients with possible hypertension: A systematic review and metaanalysis. JAMA Internal Medicine 179(3): 351-362.

Rula EY, Hobgood A (2010). The impact of health risk awareness on employee risk levels. American Journal of Health Behavior 34(5): 532-543.

Soler RE, Griffith M, Hopkins DP, Leeks KD (2009) The assessment of health risks with feedback. Results of a systematic review. In NP Pronk (Eds.), ACSM's Worksite Health Handbook: A Guide to Building Healthy and Productive Companies, pp. 82-91. Champagne: Human Kinetics.

Whelton P, Carey RM, Aronow WS, Casey Jr. DE, Collins KJ, Himmelfarb CD, et al. (2018) 2017 ACC/AHA/AAPA/ABC/ACPM/AGS/APhA/ASH/ASPC/NMA/ PCNA Guideline for the Prevention, Detection, Evaluation, and Management of High Blood Pressure in Adults. A Report of the American College of Cardiology/American Heart Association Task Force on Clinical Practice Guidelines. Hypertension 71(6). DOI: 10.1161/HYP.0000000000000065.

Williams B, Mancia G, Spiering W, Rosei EA, Azizi M, Burnier M, et al. (2018) 2018 ESC/ESH Guidelines for the management of arterial hypertension. European Heart Journal 39(33): 3021-3104.

Woringer M, Nielsen J, Zibarras L, Evason J, Kassianos A, Harris M (2017) Development of a questionnaire to evaluate patients' awareness of cardiovascular disease risk in England's National Health Service Health Check preventive cardiovascular programme. BMJ Open 7(9): e014413. 